\title{
Hematuria durante la gestación debida a tumor vesical. Presentación de 2 casos
}

\author{
A. Hernández Castrillo*, A. Villanueva Peña*, E. de Diego Rodríguez*, A. Colina Martín**, \\ J.M. Corral Mones***
}

*Servicio de Urología. ${ }^{* *}$ Servicio de Ginecología y Obstetricia. ${ }^{* * *}$ Servicio de Anatomía Patológica. Hospital Comarcal de Laredo. Cantabria.

Actas Urol Esp 2005; 29 (10): 981-984

\section{RESUMEN}

HEMATURIA DURANTE LA GESTACIÓN DEBIDA A TUMOR VESICAL. PRESENTACIÓN DE 2 CASOS

Los tumores vesicales son raros durante la gestación. Presentamos dos casos, en ambos la sintomatología inicial fue hematuria macroscópica, aunque uno de ellos fue inicialmente confundido como sangrado vaginal. Las pacientes tenían una edad de 41 y 27 años, con una edad gestacional de 26 y 18 semanas respectivamente. Los diagnósticos fueron hechos con cistoscopia y ecografía. Se utilizaron agentes tocolíticos. Se practicó resección transuretral de ambos tumores, empleándose una anestesia raquídea. No hubo efectos adversos sobre la gestación. El diagnóstico anatomopatológico fue papiloma invertido en el primer caso y neoplasia urotelial papilar de bajo potencial maligno en el segundo. Se revisa la literatura.

Palabras clave: Neoplasia urotelial papilar de bajo potencial maligno. Papiloma invertido. Tumor vesical. Resección transuretral. Gestación. Hematuria.

\section{ABSTRACT}

HEMATURIA DURING PREGNANCY CAUSED BY BLADDER TUMOUR. REPORT OF 2 CASES

Tumours of the urinary bladder are rare in pregnancy. We report two such cases presenting with gross hematuria, but one of them was initially mistaken as vaginal bleeding. Patients age was 41 and 27 years old, at 26 and 18 weeks of gestation respectively. Diagnoses were made with cystoscopy and sonography. Tocolytics agents were employed. Both of the tumours were managed succesfully by transurethral electroresection. Raquianesthesia was employed in both. There were no adverse effects on the pregnacies. Transurethral resection is a safe procedure during pregnancy. The pathology diagnoses were inverted papilloma in the first case and papillary urothelial neoplasm of low malignant potential in the other one. The literature is reviewed.

Keywords: Papillary urothelial neoplasm of low malignant potential. Inverted papilloma. Bladder tumour. Transurethral resection. Pregnancy. Hematuria.

$\mathrm{L}^{2}$ a presencia de un tumor vesical durante el embarazo es un hecho poco habitual que plantea problemas diagnósticos y terapéuticos ${ }^{1,2}$. Los tumores vesicales durante la gestación pueden simular cistitis o sangrados obstétricos ${ }^{1,2}$. Una vez hecho el diagnóstico, el tratamiento ha de ser el más eficaz y seguro, tanto para la madre como para el feto. La colaboración con anestesistas y obstetras es necesaria para el correcto manejo de esta patologia. Presentamos dos casos ocurridos en nuestro hospital y se revisa la literatura al respecto.

\section{CASOS CLÍNICOS}

Caso Clínico 1

Año 1996. paciente de 41 años, primipara gestante de 26 semanas. Alérgica a la penicilina y fumadora ocasional. Realizó primeras consultas de seguimiento de la gestación dentro de la normalidad, analítica urinaria normal.

Acudió a urgencias de nuestro hospital por hematuria, la cual cedió espontáneamente, la paciente fue remitida a consulta de urología para estudio. Se realizó cistoscopia encontrando una 
tumoración vegetante de un centímetro de diámetro en el cuello vesical. Se decidió intervención quirúrgica.

Como tocolítico su obstetra le pautó tratamiento con Ritodrina (Prepar ${ }^{\mathrm{R}}$ ). Bajo anestesia raquídea se practicó resección transuretral de lesión, dejando sonda de lavado durante 24 horas. El resto de la gestación transcurrió sin problema, concluyendo mediante cesárea programada por razones obstétricas en las 42 semanas de gestación.

El diagnóstico anatomopatológico fue de papiloma invertido (Fig. 1). Se siguió a la paciente en consulta, con cistoscopias anuales durante 5 años, sin recidiva.

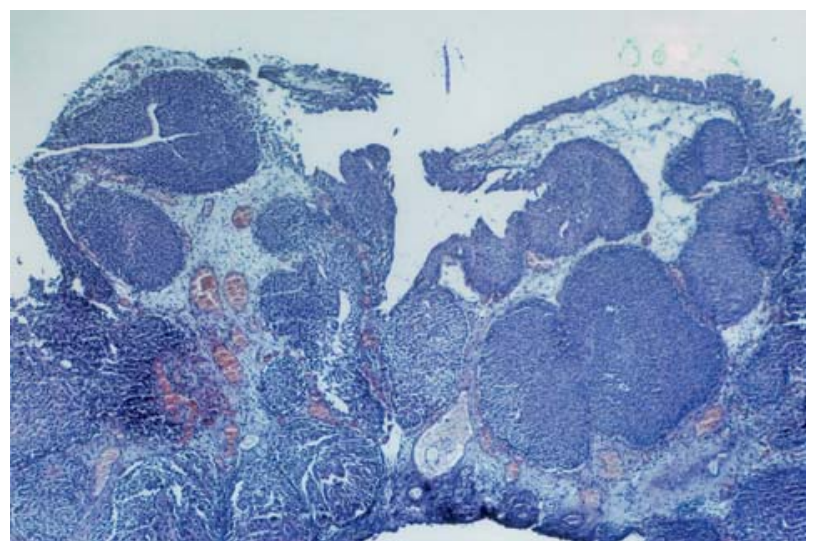

FIGURA 1. Imagen microscópica a 40 aumentos. Papiloma invertido.

\section{Caso Clínico 2}

Paciente de 27 años con antecedente de cesárea en el año 2000 y fumadora ocasional. En diciembre de 2003 se le realizó urocultivo siendo positivo para E. Coli, pautándosele tratamiento. Ese mismo mes presentó sangrado vaginal durante dos días.

En febrero de 2004, gestante de 16 semanas, acudió a urgencias por sangrado vaginal y dolor hipogástrico. Refirió haber tenido sangrado "como una regla” con grumos negros. Fue explorada por el ginecólogo de guardia, presentaba genitales externos normales, vagina normal sin restos de sangre, cérvix cerrado, útero relajado, latido fetal presente.

Se practicó ecografía obstétrica: feto vivo, biometría de 15 semanas, placenta posterior normoinserta. en vejiga parecía existir una tumora- ción (Fig. 2). Se realizó ecografía abdominal que informó de lesión endovesical de 17x28x29mm (Fig. 3). Se confirmó que lo que la paciente presentaba era hematuria macroscópica, la cual continuó hasta el día de la operación. Urocultivo negativo. Se realizó cistoscopia la cual confirmó la presencia de una tumoración de aspecto papilar de unos 3 centrímetros de diámetro, muy pediculada, en trígono, junto a meato ureteral derecho.

Se pautó tratamiento tocolítico con indometacina supositorios y profilaxis antibiótica con fosfomicina vía oral. Se realizó resección transuretral de la lesión bajo anestesia raquídea, dejando sonda de lavado durante 24 horas.

El diagnóstico anatomopatológico fue de neoplasia papilar urotelial de bajo potencial maligno (Fig. 4). El resto de la gestación transcurrió con

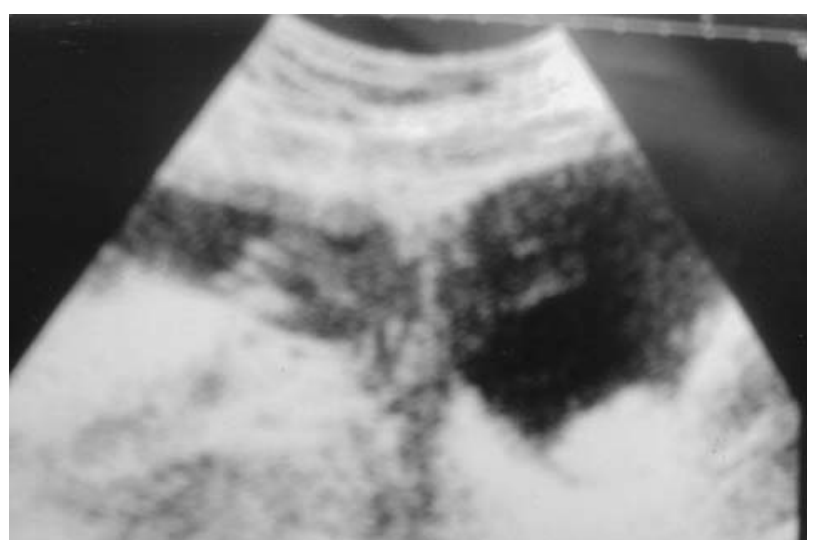

FIGURA 2. Ecografía. A la derecha se puede apreciar la vejiga con el tumor en la parte más inferior, a la izquierda se sitúa el feto.

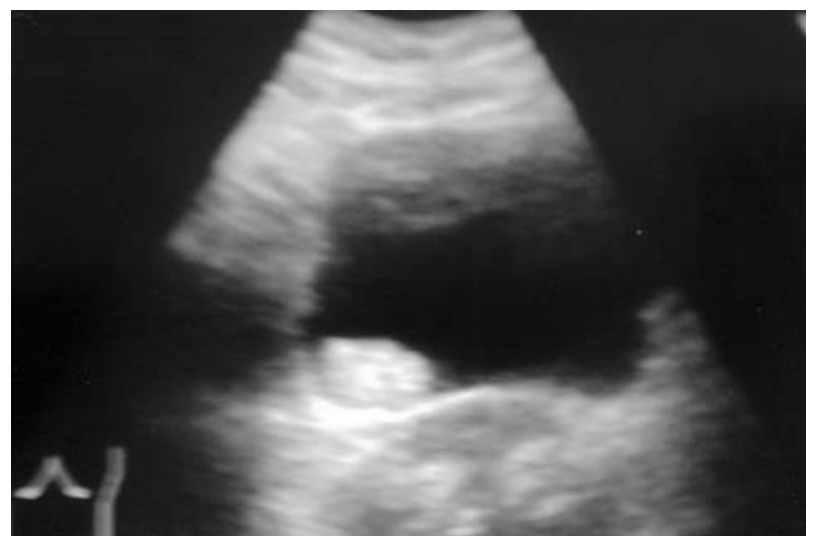

FIGURA 3. Se puede apreciar el tumor vesical situado en la base vesical derecha, en el trigono junto al orificio ureteral. 


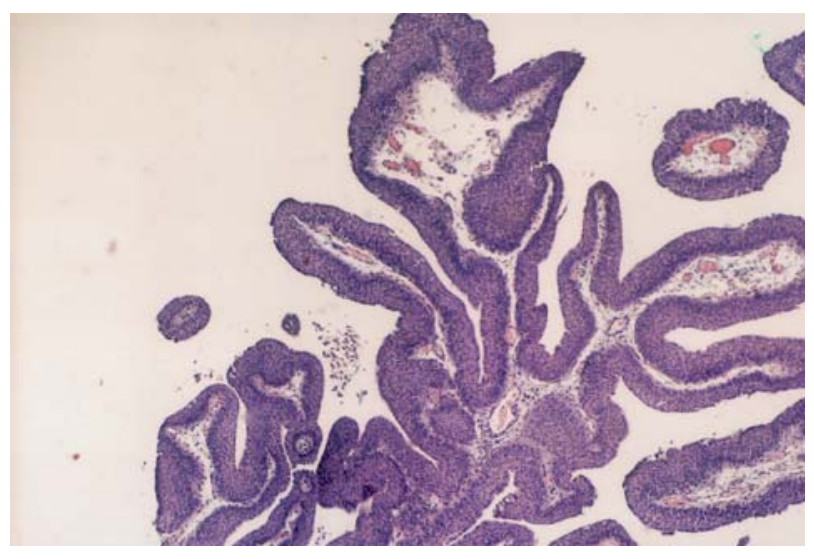

FIGURA 4. Imagen microscópica a 40 aumentos. Neoplasia urotelial papilar de bajo potencial maligno.

normalidad concluyendo a término, mediante cesárea por motivos obstétricos. Seguimiento con cistoscopias, sin recidiva hasta la actualidad.

\section{DISCUSIÓN}

Hay muy pocos casos publicados de tumores vesicales durante la gestación. Loughlin ${ }^{1}$ atribuye a Waser el primer caso comunicado, en 1927. En una revisión de la literatura en lengua inglesa, Wax ${ }^{2}$ refiere 24 casos publicados desde 1964 hasta 2001. Nosotros sólo hemos encontrado una publicación en español al respecto ${ }^{3}$.

La presencia de tumor vesical durante la gestación es poco habitual, sin embargo, conviene tener presente esta posibilidad diagnóstica.

El signo clínico más frecuente, $50 \%$ de los casos, es la hematuria macroscópica ${ }^{1,2}$. La presentación de la paciente refiriendo sangrado vaginal $\left(29 \%\right.$ de los casos según la revisión de $\left.\mathrm{Wax}^{2}\right)$ y dolor abdominal, pueden ser confundidos con causas obstétricas, como ocurrió en nuestro segundo caso. En estos casos dudosos parece razonable cateterizar la vejiga para descartar la hematuria $^{1,2}$. El tumor también puede presentarse simulando una cistitis recurrente, o una litiasis, con disuria, urgencia, polaquiuria. Incluso se ha comunicado un caso de presentación como rotura vesical durante el parto ${ }^{4}$. El diagnóstico se puede hacer mediante cistoscopia o ecografia. A veces la ecografía obstétrica de control en pacientes asintomáticas descubre el tumor ${ }^{5}$.

Tras el diagnóstico de tumor vesical, el primer paso terapéutico es la resección transuretral vesical, la cual puede ser curativa en el caso de tumores superficiales, o el paso previo a otros planteamientos terapéuticos en caso de tumor infiltrante. La RTU es un procedimiento que se ha demostrado seguro durante la gestación ${ }^{1,2}$. Sin embargo, existe preocupación sobre si la estimulación eléctrica pudiera desencadenar contracciones uterinas ${ }^{5}$. A veces la cirugía puede ser retrasada hasta después del parto, como propugnaba Soloway ${ }^{6}$ en un comentario editorial tras la comunicación de González-Blanco ${ }^{5}$. Lingan ${ }^{7}$ comunica un caso diagnosticado dos semanas después del parto, asintomático hasta entonces. Sin embargo en el caso comunicado por $\mathrm{Koko}^{4}$, la rotura de una vejiga neoplásica pudiera haber sido evitada de haber sido diagnosticado y tratado el tumor antes del parto. En nuestro segundo caso, la gestante se mantuvo con hematuria macroscópica hasta la intervención quirúrgica, en esta circunstancia es dificil retrasar la cirugía.

Cuando se plantea una cirugia en pacientes gestantes, es fundamental una buena coordinación entre anestesistas, obstetras y urólogos, en aras a una tratamiento lo más seguro posible para la madre y el feto.

En nuestro hospital se emplean tocolíticos en todas las gestantes que van a ser sometidas a cirugía abdominal, pues existe riesgo de parto pretérmino $^{8}$, aunque existe cierta controversia sobre su eficacia ${ }^{9}$. No hemos hallado referencia sobre si se emplearon tocolíticos o no en los artículos revisados sobre RTU durante la gestación, ni en las revisiones al respecto. El parto pretérmino es la principal causa de morbilidad y mortalidad neonatal, con secuelas como parálisis cerebral o enfermedad pulmonar crónica. El tocolítico se elige de acuerdo con el obstetra y la edad gestacional. En nuestro hospital se utiliza indometacina hasta las 24 semanas de gestación. Desde la semana 24 de gestación hasta la 36 , se utilizaba Ritodrine (Prepar ${ }^{\mathrm{R}}$ ), beta-mimético no exento de efectos secundarios ${ }^{9}$. Por lo que ha sido sustituido en nuestro hospital por el Tractocile (Atosiban ${ }^{\mathrm{R}}$ ), un nuevo fármaco antagonista selectivo de la oxitocina. La monitorización fetal en el perioperatorio debería quedar reflejada en la historia ${ }^{10}$. No tuvimos problemas durante la cirugía, pero hay que recordar que las $2 \mathrm{RTU}$ fueron de corta duración y sobre tumores de pequeño tamaño. Sin embargo no hemos encon- 
trado referencia en la literatura consultada a ningún problema en RTUs en pacientes gestantes. Núñez Mora comunica una RTU de 33 gramos de un leiomioma vesical en una gestante de 8 sema$\operatorname{nas}^{11}$.

Wax $^{2}$ en su revisión de 24 casos, comunican que el $74 \%$ de los tumores vesicales durante la gestación son carcinomas de células transicionales, el 22\% adenocarcinomas, y el 4,5\% carcinomas de células escamosas. En esta revisión, entre los carcinomas de células transicionales, la mayoría (79\%), son no invasivos, y el 40\%, 35\% y $25 \%$ respectivamente son de grados 1,2 y 3 . Existe un caso comunicado de carcinoma en estadio metastásico ${ }^{12}$.

En nuestros casos, el diagnóstico anatomopatológico fue de papiloma invertido y neoplasia urotelial papilar de bajo potencial maligno.

\section{REFERENCIAS}

1. Loughlin KR. The management of urological malignancies during pregnancy. Br J Urol 1995 nov;76(5):639-644.

2. Wax JR, Pinette MG, Blackstone J, Cartin A, McCrann DJ. Nonbilharzial bladder carcinoma complicating pregnancy: review of the literature. Obstet Gynecol Surv 2002 apr; 57(4):236-244.

3. Fernández Borrel A, Peinado Ibarra F, Gómez-Sancha F, Teba del Pino F, Arellano Gañán R, Pereira Sanz I. Tumor vesical durante el embarazo. Actas Urol Esp 1999 feb;23(2): 164-166.
4. Koko AH, Onuora VC, Al Shabani NA, Meabed AH, Al Turki $\mathrm{M}$, Aljawini N. Rupture of the urinary bladder during labour: an unusual presentation of bladder tumour. Br J Urol 1999 feb;83(3):348-349.

5. González-Blanco S, Mador DR, Vickar DB, McPhee MS. Primary bladder carcinoma presenting during pregnancy in 3 cases. J Urol 1989;141:613-614.

6. Soloway MS. Editorial Comment J Urol 1989;141:614.

7. Lingan K, Buckley JF, Scott R. Transitional cell carcinoma of the bladder presenting during pregnancy. $\mathrm{Br} \mathrm{J}$ Urol 1993;71:101.

8. Mazze RI, Kallen B. Appendectomy during pregnancy: a Swedich registry study of cases. Obstet Gynecol. 1991 jun; 77(6):835-840.

9. Berkman ND, Thorp JM, Jr, Lohr KN, et al. Tocolytic treatment for the management of preterm labor: a review of the evidence. Am J Obstet Gynecol 2003 jun;188(6): 1648-1659.

10. Melnick DM, WL Wahl, Dalton VK. Management of general surgical problems in the pregnant patient. Am J Surg 2004; 187:170-180.

11. Núñez Mora C, Julve Villalta E, Hardisson Hernáez D, Jiménez de León, J, Picazo García, ML, Hidalgo Togores L, et al. Leiomioma vesical durante el embarazo. Arch Esp de Urol 1999;52(5):510-512.

12. Danisman A, Kukul E, Guntekin E. Primary metastatic bladder carcinoma during pregnancy. Br J Urol 1997;79: 489.

Dr. A. Hernández Castrillo

La Concha, 99 B

39690 Villaescusa (Cantabria)

a.h.castrillo@terra.es

(Trabajo recidibo el 10 noviembre 2004) 